some urban and suburban areas fox density may be much higher (7-10 foxes $\mathrm{km}^{-2}$ ). The equation shows clearly that the required degree of vaccine-induced herd immunity is greater in dense fox populations than in sparse ones; for $K \simeq 2$ foxes $\mathrm{km}^{-2}$ the required degree of herd immunity is predicted to be approximately 80 per cent $\left(K_{\mathrm{T}}=0.4 \mathrm{~km}^{-2}\right)$. In urban areas with dense populations for example, $(K=$ $7 \mathrm{~km}^{-2}$ ) the required level of protection is predicted to be roughly 95 per cent. These figures are depressingly high and question the practical feasibility of mass vaccination as a means of control.

A further problem is created by the demography of the reservoir host species. The fox has a high fecundity (an average birth rate of $4-5$ cubs per litter each year) and a short life expectancy (between $1.5-2.5$ years from birth). These observations imply that a high proportion of fox populations are young animals in their first or second year of life. As such the maintainance of high levels of herd immunity would require repeated (preferably annual) cohort immunization with the aim of exposing young foxes to inoculated baits at as young an age as is practically possible.

The development of a safe wildlife vaccine as described by Blancou et al. is clearly an important step towards the control of rabies. In practice it will undoubtedly be of value to block transmission in areas of low fox density (given that the vaccine can be produced and delivered cheaply). But in areas of high fox density, such as many urban areas in Europe (where the risk of transmission to man and his domestic animals is greatest), mass vaccination will probably have to be supplemented by culling to reduce the target level of immunization (the degree of herd immunity) required to prevent rabies persistence.

The new vaccine's greatest value may well be in the immunization of domestic animals and pets as opposed to wildlife reservoir species. In Latin America, Asia and Africa, for example, rabies in dogs presents the major threat to man (most of the estimated 15,000 annual human deaths caused by rabies occur in these regions). In densely populated urban areas, with large free-roaming dog and cat populations, baits inoculated with a safe recombinant vaccine is potentially a very attractive method of reducing the risk of transmission to man.

Roy M. Anderson is Professor of Parasite Ecology in the Department of Pure and Applied Biology at Imperial College, University of London, London SW7 $2 B B, U K$.

\title{
History of chemistry
}

\section{Carl Scheele (1742-1786) and the discovery of oxygen}

\section{from E. L. Scott}

THE birthday of oxygen is generally taken to be 1 August 1774, the day on which Joseph Priestley used a twelve-inch lens to focus the Sun's rays on the red calx of mercury $(\mathrm{HgO})$, thereby liberating a gas in which "a candle burned... with a remarkably vigorous flame" (Priestley, J. in Experiments and Observations on Different Kinds of Air Vol. ii, 34; Johnson, London, 1775). But at least two years before that the Swedish chemist Carl Wilhelm Scheele, who died two hundred years ago, had already isolated oxygen. Moreover, Scheele's discovery was a confirmation of his belief in the existence of the gas, whereas Priestley's discovery was

$\begin{array}{lll}\text { Metal - phlogiston } & \longrightarrow & \text { calx } \\ \text { Non-metal - phlogiston } & \longrightarrow & \text { acid } \\ \text { Metal + oxygen } & \longrightarrow & \text { basic oxide } \\ \text { Non-metal + oxygen } & \longrightarrow & \text { acidic oxide }\end{array}$

A comparison of the phlogiston and oxygen theories of combustion. Lavoisier, like his predecessors, did not distinguish between an acidic oxide and the acid which it forms on combining with water. totally unexpected.

Scheele's experiments and conjectures are described in his only book, Chemische Abhandlung von der Luft und dem Feuer (Uppsala and Leipzig, 1777), translated into English in 1780 (Scheele, C.W. Chemical Observations and Experiments on Air and Fire (trans. Forster, J.R.) Johnson, London, 1780). Within a few years the French chemist Lavoisier named the gas oxygène, believing it to be present in all acids, and had demonstrated its role in combustion. Previously, for more than a century, burning and the calcination of metals had been explained in terms of phlogiston - a 'principle' present in all metals and inflammable non-metals. On the application of sufficient heat, the phlogiston was thought to be attracted by the surrounding air, leaving a calx in the case of a metal and an acid when the substance burned was a non-metal.

Scheele, in several experiments carried out in closed vessels observed the diminution in volume of the air and the failure of the residual air to support combustion, processes now recognizable as oxidation. He concluded that air consists of two

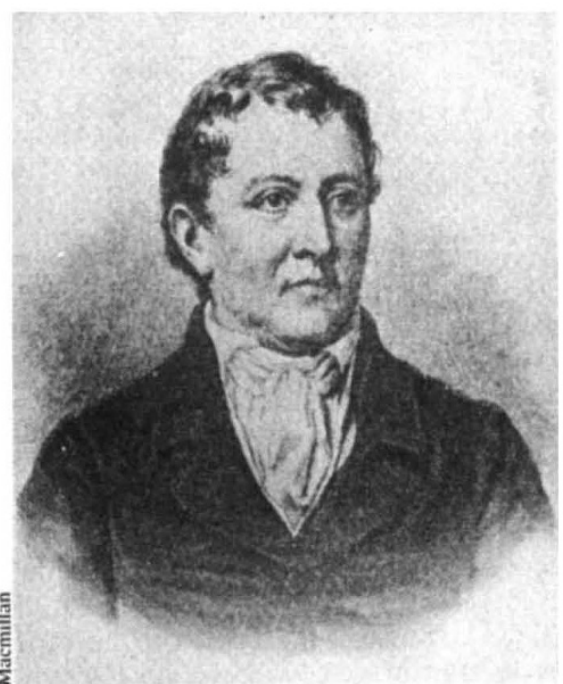

gases, only one of which (occupying about a quarter of the volume) attracts phlogiston. This latter gas he termed Feuerluft (fire air) and eventually isolated it by heating concentrated nitric acid with charcoal, absorbing in milk of lime the red fumes of nitrogen dioxide generated. The oxygen remaining was formed by the thermal decomposition of the acid.

Scheele came close to anticipating Lavoisier by considering the possibility that during combustion fire air combines with the burning body, but he eventually concluded that the gas unites with phlogiston forming heat - which, like most of his contemporaries, he regarded as a material. This hypothesis enabled Scheele to explain why, in a few cases, a calx could be restored to a metal by heat alone, whereas normally the presence of a substance rich in phlogiston (for example, charcoal) was necessary.

Although misled by his belief in phlogiston, Scheele's record of achievement during his short life is remarkable. He discovered radiant heat, noting the similarity of its properties to those of light. His investigation of fluorite, pyrolusite and molybdenite led, respectively, to the discoveries of hydrofluoric acid, chlorine and molybdenum. He discovered glycerol and isolated a dozen previously unknown organic acids. A minor mystery surrounds his discovery of the toxic hydrocyanic acid, resulting from his investigation of the pigment Prussian blue, for he described its taste and smell.

From the age of fifteen Scheele worked in various pharmacies in Sweden until, in the summer of 1775 , he acquired his own pharmacy from the widow of Herman Pohl in the small town of Köping. Though subsequently offered academic posts he refused to leave his practice. A few days before his death in 1786 he married Pohl's widow and made her his heiress.

E. L Scott is a retired schoolmaster and historian at Spring Hill, Careby, Stamford, Lincolnshire PE9 4EA, UK. 